Document downloaded from:

http://hdl.handle.net/10251/64679

This paper must be cited as:

Behl, R.; Cordero Barbero, A.; Motsa, SS.; Torregrosa Sánchez, JR. (2015). Construction of fourth-order optimal families of iterative methods and their dynamics. Applied Mathematics and Computation. 271:89-101. doi:10.1016/j.amc.2015.08.113.

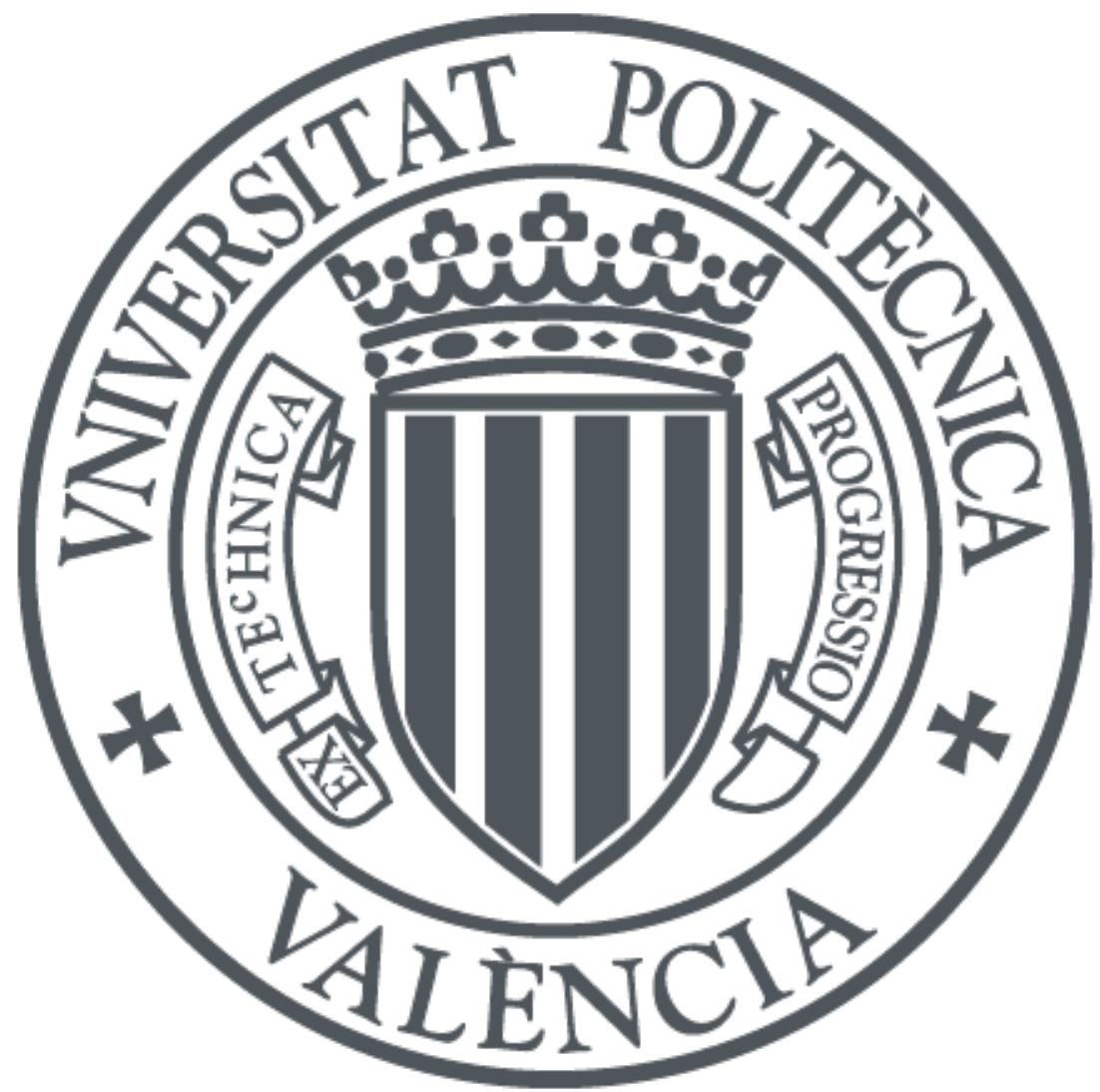

The final publication is available at

https://dx.doi.org/10.1016/j.amc.2015.08.113

Copyright Elsevier

Additional Information 


\title{
Construction of fourth-order optimal families of iterative methods and their dynamics 放
}

\author{
Ramandeep Behl ${ }^{\mathrm{a}}$, Alicia Cordero ${ }^{\mathrm{b}, *}$, Sandile S. Motsa ${ }^{\mathrm{a}}$, Juan R. Torregrosa ${ }^{\mathrm{b}}$ \\ ${ }^{a}$ School of Mathematical Sciences, University of KwaZulu-Natal, Private Bag X01, Scottsville 3209, Pietermaritzburg, \\ South Africa \\ ${ }^{b}$ Instituto Universitario de Matemática Multidisciplinar, Universitat Politècnica de València, Cno. de Vera s/n, \\ Valencia, Spain
}

\begin{abstract}
In this paper, we propose a general class of fourth-order optimal multi-point methods without memory for obtaining simple roots. This class requires only three functional evaluations (viz. two evaluations of function $f\left(x_{n}\right), f\left(y_{n}\right)$ and one of its first-order derivative $\left.f^{\prime}\left(x_{n}\right)\right)$ per iteration. Further, we show that the well-known Ostrowski's method and King's family of fourth-order procedures are special cases of our proposed schemes. One of the new particular subclasses is a biparametric family of iterative methods. By using complex dynamics tools, its stability is analyzed, showing stable members of the family. Further on, one of the parameters is fixed and the stability of the resulting class is studied. On the other hand, the accuracy and validity of new schemes is tested by a number of numerical examples by comparing them with recent and classical optimal fourth-order methods available in the literature. It is found that they are very useful in high precision computations.
\end{abstract}

Keywords: Nonlinear equations, Iterative methods, Optimal order of convergence, Complex dynamics, Parameter plane, Basin of attraction.

\section{Introduction}

The conceptualization and construction of multi-point methods for finding efficient and accurately the approximate solution $\xi$ of nonlinear equation $f(x)=0$, is an essential task in numerical analysis. This topic has attracted the attention of many researchers, when Traub [1] initiated the analysis of these methods. Very recently, Petković et al. 2] gather up and updated the state of the art of multi-point methods. The advantage of multi-point methods is that they do not use higher order derivatives and has great practical importance because they overcome the theoretical limitations of one-point methods regarding their convergence order and computational efficiency.

As the order of an iterative method increases, so does the number of functional evaluations per step. The efficiency index [3] gives a measure of the balance between those quantities, according to the formula $p^{\frac{1}{d}}$, where $p$ is the order of convergence of the method and $d$ the number of functional evaluations per iteration. According to the Kung-Traub conjecture [4, the order of convergence of any multi-point method cannot exceed the bound $2^{n-1}$, called the optimal order.

In the past, Ostrowski was the first mathematician who proposed an optimal fourth-order multi-point method which requires only three functional evaluations. On the other hand, Jarratt [5, 6], in 1966 and King [7, in 1975, proposed various optimal fourth-order multi-point methods. King further showed that Ostrowski's method was a particular case of his family.

With the advancement of computer algebra, many researchers like Chun [8, Chun and Ham [9], Cordero et al. [10, Sharma and Ghua [11, Kanwar et al. [12, Sharifi et al. [13, Soleymani et al. [14]

\footnotetext{
This research was partially supported by Ministerio de Economía y Competitividad MTM2014-52016-C02-02

* Corresponding author

Email addresses: ramanbeh187@yahoo.in (Ramandeep Behl), acordero@mat.upv.es (Alicia Cordero ), sandilemotsa@gmail.com (Sandile S. Motsa), jrtorre@mat.upv.es (Juan R. Torregrosa)
} 
and Behl et al. [15], among others, proposed various optimal schemes or families of methods of order four. But Ostrowski', Jarratt' and King's methods are between the most efficient fourth-order methods known to date. Therefore, obtaining new optimal methods of order four is still important, because they combine high order of convergence and low computational cost.

The main aim of this manuscript is to suggest and analyze a new general class of fourth-order optimal methods for solving nonlinear equations. In terms of computational cost, they require only three functional evaluations per full iteration. We demonstrate the usefulness of the proposed method by performing several numerical examples and observe that our methods are equal and better competent than the existing classical methods and recent robust methods that are available in the literature.

On the other hand, we analyze the complex dynamical behavior of this family in order to select those elements of the class with better stability properties. In order to get this aim, we recall some concepts of complex dynamics that we use in this paper, and can be completed in [16]. Let us assume that a fixed point iteration function acts on an arbitrary polynomial $p(z)$; that yields a rational function, that will be denoted by $R$. So, given a rational function $R: \hat{\mathbb{C}} \rightarrow \hat{\mathbb{C}}$, where $\hat{\mathbb{C}}$ is the Riemann sphere, the orbit of a point $z_{0} \in \hat{\mathbb{C}}$ is defined as:

$$
\left\{z_{0}, R\left(z_{0}\right), R^{2}\left(z_{0}\right), \ldots, R^{n}\left(z_{0}\right), \ldots\right\}
$$

We analyze the phase plane of the map $R$ by classifying the starting points from the asymptotical behavior of their orbits. A $z_{0} \in \hat{\mathbb{C}}$ is called a fixed point if $R\left(z_{0}\right)=z_{0}$. A periodic point $z_{0}$ of period $p>1$ is a point such that $R^{p}\left(z_{0}\right)=z_{0}$ and $R^{k}\left(z_{0}\right) \neq z_{0}$, for $k<p$. A pre-periodic point is a point $z_{0}$ that is not periodic but there exists a $k>0$ such that $R^{k}\left(z_{0}\right)$ is periodic. A point $z_{0}$ is a critical point of the rational map $R$ if $R$ fails to be injective in any neighborhood of $z_{0}$. Indeed, if a critical point is different from those associated with the roots of the polynomial $p(z)$, it is called free critical point.

Moreover, a fixed point $z_{0}$ is called attractor if $\left|R^{\prime}\left(z_{0}\right)\right|<1$, superattractor if $\left|R^{\prime}\left(z_{0}\right)\right|=0$, repulsor if $\left|R^{\prime}\left(z_{0}\right)\right|>1$ and parabolic if $\left|R^{\prime}\left(z_{0}\right)\right|=1$. The fixed points different from those associated with the roots of the polynomial $p(z)$ are called strange fixed points.

The basin of attraction of an attractor $\alpha$ is defined as:

$$
\mathcal{A}(\alpha)=\left\{z_{0} \in \hat{\mathbb{C}}: R^{n}\left(z_{0}\right) \rightarrow \alpha, n \rightarrow \infty\right\}
$$

The Fatou set of the rational function $R, \mathcal{F}(R)$, is the set of points $z \in \hat{\mathbb{C}}$ whose orbits tend to an attractor (fixed point or periodic orbit). Its complement in $\hat{\mathbb{C}}$ is the Julia set, $\mathcal{J}(R)$. That means that the basin of attraction of any fixed or periodic point belongs to the Fatou set and the boundaries of these basins of attraction belong to the Julia set.

The rest of the paper is organized as follows: in Section 2 the optimal class of methods is presented and its local order of convergence is analyzed. Some especial cases of the proposed family are described in Section 3. One bi-parametric class from those appearing in Section 3 is studied under the point of view of complex dynamics. Some numerical test are performed in Section 4 and, finally, some conclusions are stated.

\section{Development of our optimal scheme}

In this section, we propose a new general optimal class of fourth-order multi-point methods. By using a similar idea to that presented by Chun in [8] and by Artidiello in [17, we consider the following scheme

$$
\left\{\begin{array}{c}
y_{n}=x_{n}-\frac{f\left(x_{n}\right)}{f^{\prime}\left(x_{n}\right)} \\
x_{n+1}=x_{n}-\frac{f\left(x_{n}\right)}{f^{\prime}\left(x_{n}\right)} H\left(u\left(x_{n}\right)\right)
\end{array}\right.
$$

where $u\left(x_{n}\right)=\frac{f\left(x_{n}\right)}{f\left(x_{n}\right)+\alpha f\left(y_{n}\right)}$ and $H(u)$ is a real-valued weight function, such that its order of convergence reaches at the optimal level fourth without using any more functional evaluations. Theorem 2.1 indicates under what conditions on the weight function in (2.1), the order of convergence will reach the optimal level four. 
Theorem 2.1. Let $f$ be a sufficiently smooth function $f: D \subseteq \mathbb{R} \rightarrow \mathbb{R}$ with a simple zero $\xi$ in the open interval D. Let $H(u)$ be a real-valued differentiable function. If an initial approximation $x_{0}$ is sufficiently close to the required root $\xi$ of function $f$, then the convergence order of our scheme (2.1) is equal to four when function $H$ satisfies the following conditions

$$
H(1)=1, \quad H^{\prime}(1)=-\frac{1}{\alpha}, \quad H^{\prime \prime}(1)=\frac{2 \alpha+4}{\alpha^{2}}, \text { where } \alpha \neq 0 .
$$

In this case, the error equation is

$$
e_{n+1}=\left(\alpha^{2}+4 \alpha+5+\frac{\alpha^{3}}{6} H^{\prime \prime \prime}(1)\right) e_{n}^{4}+O\left(e_{n}^{5}\right)
$$

where $H^{\prime \prime \prime}(1)$ is any finite quantity, $e_{n}=x_{n}-\xi$ and $c_{k}=\frac{1}{k !} \frac{f^{(k)}(\xi)}{f^{\prime}(\xi)}, \quad k=2,3,4, \ldots$

Proof: Let $\xi$ be a simple zero of $f(x)$. Expanding $f\left(x_{n}\right)$ and $f^{\prime}\left(x_{n}\right)$ about $x=\xi$ by Taylor series expansion, we have

$$
f\left(x_{n}\right)=f^{\prime}(\xi)\left(e_{n}+c_{2} e_{n}^{2}+c_{3} e_{n}^{3}+c_{4} e_{n}^{4}+O\left(e_{n}^{5}\right)\right)
$$

and

$$
f^{\prime}\left(x_{n}\right)=f^{\prime}(\xi)\left(1+2 c_{2} e_{n}+3 c_{3} e_{n}^{2}+4 c_{4} e_{n}^{3}+O\left(e_{n}^{4}\right)\right)
$$

respectively.

From equations 2.4 and $(2.5)$, we get

$$
\frac{f\left(x_{n}\right)}{f^{\prime}\left(x_{n}\right)}=e_{n}-c_{2} e_{n}^{2}+2\left(c_{2}^{2}-c_{3}\right) e_{n}^{3}+\left(-4 c_{2}^{3}+7 c_{2} c_{3}-3 c_{4}\right) e_{n}^{4}+O\left(e_{n}^{5}\right),
$$

and in combination with Taylor series expansion of $f\left(x_{n}-\frac{f\left(x_{n}\right)}{f^{\prime}\left(x_{n}\right)}\right)$ about $x=\xi$, we obtain

$$
f\left(y_{n}\right)=f\left(x_{n}-\frac{f\left(x_{n}\right)}{f^{\prime}\left(x_{n}\right)}\right)=f^{\prime}(\xi)\left[c_{2} e_{n}^{2}+\left(-2 c_{2}^{2}+2 c_{3}\right) e_{n}^{3}+\left(5 c_{2}^{3}-7 c_{2} c_{3}+3 c_{4}\right) e_{n}^{4}+O\left(e_{n}^{5}\right)\right] .
$$

Therefore, we have

$$
\begin{aligned}
u\left(x_{n}\right)=\frac{f\left(x_{n}\right)}{f\left(x_{n}\right)+\alpha f\left(y_{n}\right)} & =1-\alpha c_{2} e_{n}+\alpha\left((3+\alpha) c_{2}^{2}-2 c_{3}\right) e_{n}^{2}-\alpha\left\{\left(8+6 \alpha+\alpha^{2}\right) c_{2}^{3}-2(5+2 \alpha) c_{2} c_{3}\right. \\
& \left.+3 c_{4}\right\} e_{n}^{3}+O\left(e_{n}^{4}\right) .
\end{aligned}
$$

It is clear from $(2.8)$ that $u=1+v$. Then, $v=u-1$ is infinitesimal with the same order of $e_{n}$. Therefore, we can expand weight function $H(u)$ in the neighborhood of 1 by Taylor series expansion up to third terms as follows

$$
H(u)=H(1)+H^{\prime}(1) v+\frac{1}{2 !} H^{\prime \prime}(1) v^{2}+\frac{1}{3 !} H^{\prime \prime \prime}(1) v^{3}+O\left(e_{n}^{4}\right) .
$$

By using equations $2.4-2.9$ in scheme 2.1 , we get the following error equation

$$
\begin{aligned}
e_{n+1} & =(1-H(1)) e_{n}+\left(H(1)+\alpha H^{\prime}(1)\right) c_{2} e_{n}^{2}-\left[\frac{1}{2}\left\{4 H(1)+\alpha\left(\alpha H^{\prime \prime}(1)+2(4+\alpha) H^{\prime}(1)\right)\right\} c_{2}^{2}\right. \\
& \left.-2\left(H(1)+H^{\prime}(1) \alpha\right) c_{3}\right] e_{n}^{3}-\left[\left\{7 H(1)+2 \alpha\left(7 H^{\prime}(1)+2 H^{\prime}(1) \alpha+H^{\prime \prime}(1) \alpha\right)\right\} c_{2} c_{3}+3\left(H(1)+H^{\prime}(1) \alpha\right) c_{4}\right. \\
& \left.-\frac{1}{6}\left[24 H(1)+\alpha\left\{\alpha\left(21 H^{\prime \prime}(1)+6 H^{\prime \prime}(1) \alpha+H^{\prime \prime \prime}(1) \alpha\right)+6 H^{\prime}(1)\left(13+7 \alpha+\alpha^{2}\right)\right\}\right] c_{2}^{3}\right] e_{n}^{4}+O\left(e_{n}^{5}\right) .
\end{aligned}
$$

For obtaining an iterative method of order four, the coefficients of $e_{n}, e_{n}^{2}$ and $e_{n}^{3}$ in the error equation 2.10 must be zero simultaneously. After some simplifications, we obtain equations involving 
$H(1), H^{\prime}(1), H^{\prime \prime}(1)$ and the free disposable parameter $\alpha$, which are given by

$$
\left\{\begin{array}{l}
1-H(1)=0 \\
H(1)+\alpha H^{\prime}(1)=0, \\
\frac{1}{2}\left\{4 H(1)+\alpha\left(\alpha H^{\prime \prime}(1)+2(4+\alpha) H^{\prime}(1)\right)\right\} c_{2}^{2}-2\left(H(1)+H^{\prime}(1) \alpha\right) c_{3}=0 .
\end{array}\right.
$$

After simplifying equations (2.11), we have the following conditions on the weight function

$$
H(1)=1, \quad H^{\prime}(1)=-\frac{1}{\alpha}, \quad H^{\prime \prime}(1)=\frac{2 \alpha+4}{\alpha^{2}} .
$$

Finally, from these conditions we get the following error equation

$$
e_{n+1}=\left(\alpha^{2}+4 \alpha+5+\frac{\alpha^{3}}{6} H^{\prime \prime \prime}(1)\right) e_{n}^{4}+O\left(e_{n}^{5}\right),
$$

where $\alpha \neq 0$ and $H^{\prime \prime \prime}(1)$ are free disposable parameters.

This reveals that our proposed scheme 2.1) reaches the optimal order of convergence four by using only three functional evaluations per full iteration. This completes the proof of the theorem.

\section{Special cases}

In this section, we consider some particular cases of the proposed scheme 2.1] depending upon the weight function $H(u)$. Some interesting selections are the following.

Case 1. First of all, we show that the well-known fourth-order Ostrowski's method is a particular case of our scheme. For this purpose, if we consider the following weight function

$$
H(u)=\frac{u(\alpha+1)-1}{u(\alpha+2)-2},
$$

then we get the well-known fourth-order Ostrowski's method, which is given by

$$
\left\{\begin{aligned}
y_{n} & =x_{n}-\frac{f\left(x_{n}\right)}{f^{\prime}\left(x_{n}\right)} \\
x_{n+1} & =x_{n}-\frac{f\left(x_{n}\right)}{f^{\prime}\left(x_{n}\right)}\left[\frac{f\left(x_{n}\right)-f\left(y_{n}\right)}{f\left(x_{n}\right)-2 f\left(y_{n}\right)}\right] .
\end{aligned}\right.
$$
2.1

Case 2. Now, we consider the following weight function which satisfies all the conditions of Theorem

$$
H(u)=\frac{2 u}{\alpha^{2}}+\frac{2+\alpha}{u \alpha^{2}}+\frac{\alpha^{2}-\alpha-4}{\alpha^{2}} .
$$

After using this weight function in scheme 2.1), we get the well-known fourth-order King's family, which is given by

$$
\left\{\begin{array}{c}
y_{n}=x_{n}-\frac{f\left(x_{n}\right)}{f^{\prime}\left(x_{n}\right)} \\
x_{n+1}=y_{n}-\frac{f\left(y_{n}\right)}{f^{\prime}\left(x_{n}\right)} \frac{f\left(x_{n}\right)+(\alpha+2) f\left(y_{n}\right)}{f\left(x_{n}\right)+\alpha f\left(y_{n}\right)} .
\end{array}\right.
$$

Case 3. Let us consider another weight function

$$
H(u)=\frac{2}{u^{2} \alpha^{2}}+\frac{\alpha-4}{u \alpha^{2}}+\frac{\alpha^{2}-\alpha+2}{\alpha^{2}},
$$

that satisfies all the conditions of Theorem 2.1. Therefore, we obtain

$$
\left\{\begin{array}{c}
y_{n}=x_{n}-\frac{f\left(x_{n}\right)}{f^{\prime}\left(x_{n}\right)} \\
x_{n+1}=y_{n}-\frac{f\left(y_{n}\right)}{f^{\prime}\left(x_{n}\right)} \frac{f\left(x_{n}\right)+2 f\left(y_{n}\right)}{f\left(x_{n}\right)} .
\end{array}\right.
$$


Case 4. Now, we consider the weight function

$$
H(u)=\frac{\alpha+2}{3 \alpha^{2}} u^{3}-\frac{2(1+\alpha)}{\alpha^{2}} u+\frac{3 \alpha^{2}+5 \alpha+4}{3 \alpha^{2}},
$$

satisfying all the conditions of Theorem 2.1. Therefore, we obtain

$$
\left\{\begin{array}{c}
y_{n}=x_{n}-\frac{f\left(x_{n}\right)}{f^{\prime}\left(x_{n}\right)}, \\
x_{n+1}=x_{n}-\frac{f\left(x_{n}\right)}{f^{\prime}\left(x_{n}\right)}\left[\frac{\alpha+2}{3 \alpha^{2}}\left(\frac{f\left(x_{n}\right)}{f\left(x_{n}\right)+\alpha f\left(y_{n}\right)}\right)^{3}-\frac{2(1+\alpha)}{\alpha^{2}} \frac{f\left(x_{n}\right)}{f\left(x_{n}\right)+\alpha f\left(y_{n}\right)}+\frac{3 \alpha^{2}+5 \alpha+4}{3 \alpha^{2}}\right] .
\end{array}\right.
$$

This is a new optimal family of fourth-order methods. For $\alpha=-2$, we get again well-known fourth-order Ostrowski's method.

Case 5. Instead of considering new weight functions, if we simply use equation $(2.2)$ in already defined weight function 2.9 then we get

$$
H(u)=1-\frac{v}{\alpha}+\frac{(\alpha+2) v^{2}}{\alpha^{2}}+\frac{1}{6} H^{\prime \prime \prime}(1) v^{3},
$$

where notation $v=u-1$ is introduced for simplicity. After using this weight function in scheme 2.1, we obtain

$$
\left\{\begin{aligned}
y_{n} & =x_{n}-\frac{f\left(x_{n}\right)}{f^{\prime}\left(x_{n}\right)} \\
x_{n+1} & =x_{n}-\frac{f\left(x_{n}\right)}{f^{\prime}\left(x_{n}\right)}\left[1+\frac{f\left(y_{n}\right)}{f\left(x_{n}\right)+\alpha f\left(y_{n}\right)}+(\alpha+2)\left(\frac{f\left(y_{n}\right)}{f\left(x_{n}\right)+\alpha f\left(y_{n}\right)}\right)^{2}-\left(\frac{\alpha f\left(y_{n}\right)}{\left.f\left(x_{n}\right)+\alpha f\left(y_{n}\right)\right)}\right)^{3} \frac{H^{\prime \prime \prime}(1)}{6}\right]
\end{aligned}\right.
$$

where $\alpha \neq 0$ and $H^{\prime \prime \prime}(1)$ are free disposable parameters. In the following, $H^{\prime \prime \prime}(1)$ will be denoted by $\beta$. We can easily develop various optimal families of fourth-order methods by fixing one disposable parameter at time.

\section{Dynamical analysis}

In this section, we use some tools from complex dynamics. So, we need that function $f$ is defined on Riemann sphere $\hat{\mathbb{C}}$. Our aim is to deep in the dynamical analysis of this two-parametric class of methods (3.10), whose fixed point operator is:

$$
\overline{M_{f}}(z, \alpha, \beta)=z-\left(1+u_{f}(z)+(\alpha+2) u_{f}(z)^{2}-\frac{\alpha^{3} \beta}{6} u_{f}(z)^{3}\right) \frac{f(z)}{f^{\prime}(z)}
$$

where $u_{f}(z)=\frac{f\left(z-\frac{f(z)}{f^{\prime}(z)}\right)}{f(z)+\alpha f\left(z-\frac{f(z)}{f^{\prime}(z)}\right)}$.

Under the point of view of complex dynamics, we study the general convergence of family 3.10 on quadratic polynomials. The following Scaling Theorem shows that the dynamics of two rational functions related by means of an affine transformation is conjugated.

Theorem 4.1 (Scaling Theorem). Let $A(z)=a z+b$ be an affine transformation. Let also $f(z)$ be a analytic function and $g(z)=(f \circ A)(z)$. Then, the associated operators to family $(3.10) \overline{M_{f}}$ and $\overline{M_{g}}$ are affinely conjugated by $A$, that is, $\left(A \circ \overline{M_{g}} \circ A^{-1}\right)(z)=\overline{M_{f}}(z)$, for all $z$.

Proof: We will prove the equivalent equality:

$$
\left(A \circ \overline{M_{g}}\right)(z)=\left(\overline{M_{f}} \circ A\right)(z), \text { for all } z \text {. }
$$


By developing the left side,

$$
\begin{aligned}
\left(A \circ \overline{M_{g}}\right)(z) & =a \overline{M_{g}}(z)+b \\
& =a z-a\left[1+u_{g}(z)+(\alpha+2) u_{g}(z)^{2}-\frac{\alpha^{3} \beta}{6} u_{g}(z)^{3}\right] \frac{g(z)}{g^{\prime}(z)}+b .
\end{aligned}
$$

Taking into account that $u_{g}(z)=u_{f}(A(z))$, the thesis is obtained.

So, the behavior of this class of methods on quadratic polynomials can be reduced to the study of these schemes applied on $p(z)=(z-a)(z-b)$ because any quadratic polynomial can be transformed into $p(z)$ by and affine map. Then, the resulting rational operator

$$
M_{p}(z, \alpha, \beta, a, b)=z+\frac{1}{a+b-2 z} p(z)\left(1-\frac{\alpha^{3} \beta p(z)^{3}}{6 q(z)^{3}}+\frac{(2+\alpha) p(z)^{2}}{q(z)^{2}}+\frac{p(z)}{q(z)}\right),
$$

where $q(z)=a^{2}+2 a b+a \alpha b+b^{2}-(4+\alpha)(a+b) z+(4+\alpha) z^{2}$, depends on parameters $\alpha, \beta, a$ and $b$. However, parameters $a$ and $b$ can be obviated by considering the conjugacy map (see [18])

$$
h(z)=\frac{z-a}{z-b}
$$

with the following properties:

$$
h(\infty)=1, \quad h(i \sqrt{a})=0, \quad h(-i \sqrt{a})=\infty .
$$

Then, operator $M_{p}(z, \alpha, \beta, a, b)$ is conjugated to

$$
\begin{aligned}
O_{p}(z, \alpha, \beta) & =\left(h \circ M_{p} \circ h^{-1}\right)(z) \\
& =z^{4} \frac{\left(\alpha^{3}(\beta+6 z)+6 \alpha^{2}(1+3 z(2+z))+6(1+z)^{2}(5+z(4+z))+6 \alpha(4+3 z(5+z(4+z)))\right)}{6+18(2+\alpha) z+6(14+3 \alpha(4+\alpha)) z^{2}+6(2+\alpha)(7+\alpha(4+\alpha)) z^{3}+\left(30+6 \alpha(4+\alpha)+\alpha^{3} \beta\right) z^{4}} .
\end{aligned}
$$

\subsection{Analysis of two-parametric operator $O_{p}(z, \alpha, \beta)$}

We analyze the dynamics of operator 4.2 in terms of parameters $\alpha$ and $\beta$, calculating firstly the fixed points of $O_{p}(z, \alpha, \beta)$ and studying their stability depending on the values of both parameters.

As fixed points satisfy $O_{p}(z, c)=z$, it can be checked that $z=0$ and $z=\infty$, associated to the roots of $p(z)$, are fixed points and also the strange fixed points $z=1$ and the roots of polynomial $6+(42+18 \alpha) z+$ $\left(126+90 \alpha+18 \alpha^{2}\right) z^{2}+\left(180+156 \alpha+48 \alpha^{2}+6 \alpha^{3}-\alpha^{3} \beta\right) z^{3}+\left(126+90 \alpha+18 \alpha^{2}\right) z^{4}+(42+18 \alpha) z^{5}+6 z^{6}$, that will be called $s_{i}(\alpha, \beta), i=1,2, \ldots, 6$.

In order to analyze the stability of the strange fixed points, we evaluate numerically the absolute value of the derivated operator at each of them (that is, we get their stability function), $\left|O_{p}^{\prime}\left(s_{i}, \alpha, \beta\right)\right|$, for a range of real values of both parameters $\alpha \in[-25,25]$ and $\beta \in[-60,60]$. In Figure 1 , the stability function of $z=1$ is presented. We observe that clear blue regions show the values of parameters that yield the fixed point to be repulsive, that is, where $\left|O_{p}^{\prime}(1, \alpha, \beta)\right|>1$.

The stability functions of $s_{1}(\alpha, \beta)$ and $s_{2}(\alpha, \beta)$ coincide, so only one of them is showed in Figure 2a. The same happens with $s_{3}(\alpha, \beta)$ and $s_{4}(\alpha, \beta)$ (see Figure $\left.2 \mathrm{~b}\right)$. However, the stability functions of $s_{5}(\alpha, \beta)$ and $s_{6}(\alpha, \beta)$ are different, as can be seen in Figure $2 \mathrm{c}$ and $2 \mathrm{~d}$.

Let us observe that there exist wide clear blue regions, common to all the figures, that correspond to elements of the biparametric family where all the strange fixed points are repulsive. So, they are good methods to be tested numerically. Nevertheless, we observe in some of the stability functions a discontinuity at $\beta=0$. In the following subsection we deep in the dynamical analysis of this subclass of methods. 


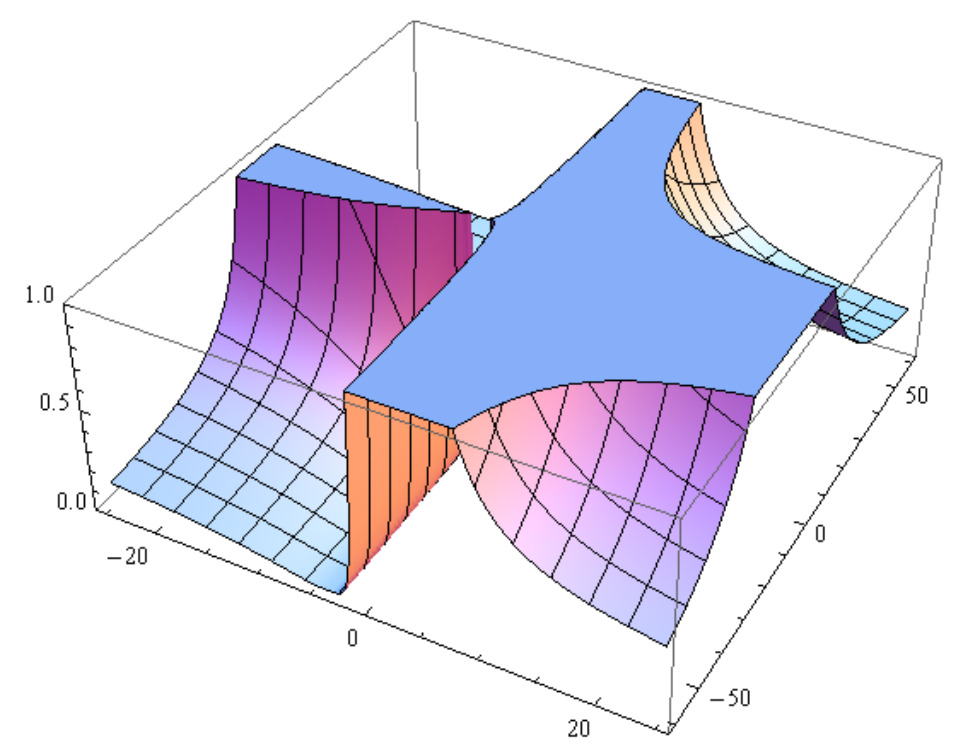

Figure 1: Stability function $\left|O_{p}^{\prime}(1, \alpha, \beta)\right|$

\subsection{The parametric family}

When $\beta=0$ is considered in 4.2 , the rational operator is simplified and takes the form

$$
O_{p}(z, \alpha, 0)=z^{4} \frac{5+z(4+z)+2(2+z) \alpha+\alpha^{2}}{1+z(4+5 z+\alpha(2+z(4+\alpha)))} .
$$

Moreover, there exist several values of parameter $\alpha$ that make operator 4.3 simpler:

- If $\alpha=-3-i$, then $O_{p}(z,-3-i, 0)=z^{4} \frac{(-1-2 i)+z}{-1+(1+2 i) z}$.

- When $\alpha=-1-i$, then $O_{p}(z,-1-i, 0)=z^{4} \frac{(z+2) i}{(2+i) z+i}$

- If $\alpha=-2$, then $O_{p}(z,-2,0)=z^{4}$ and the scheme satisfies Cayley test (see [19]). It corresponds to Ostrowski's method.

- For $\alpha=-2-\sqrt{2} i, O_{p}(z,-2-\sqrt{2} i, 0)=-z^{4} \frac{-1-2 i \sqrt{2} z+z^{2}}{-1+2 i \sqrt{2} z+z^{2}}$.

- When $\alpha=-2+\sqrt{2} i, O_{p}(z,-2+\sqrt{2} i, 0)=-z^{4} \frac{-1+2 i \sqrt{2} z+z^{2}}{-1-2 i \sqrt{2} z+z^{2}}$.

- If $\alpha=-2+2 i$, then $O_{p}(z,-2+2 i, 0)=-z^{4} \frac{-3+4 i z+z^{2}}{-1-4 i z+3 z^{2}}$.

It is specially important that there exist different specific methods with lower degree polynomials in their associated rational function, as the number of strange fixed points and free critical points is also lower. In general, the number of strange fixed points and relations between them is established in the following result. 


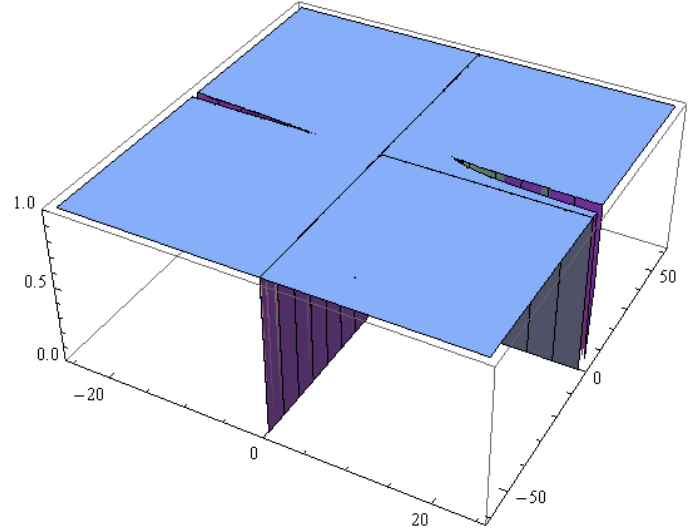

(a) $\left|O_{p}^{\prime}\left(s_{1}, \alpha, \beta\right)\right|$

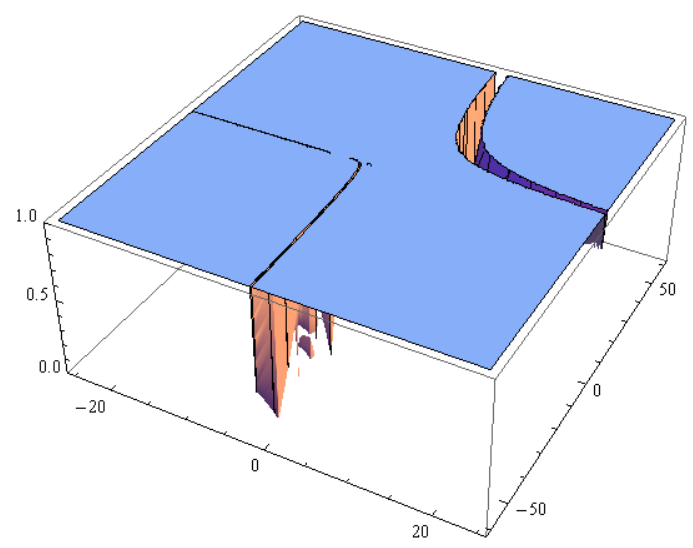

(c) $\left|O_{p}^{\prime}\left(s_{5}, \alpha, \beta\right)\right|$

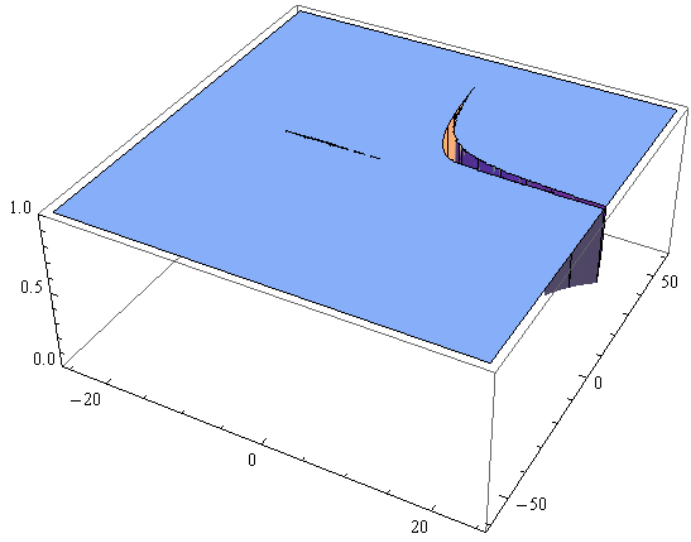

(b) $\left|O_{p}^{\prime}\left(s_{3}, \alpha, \beta\right)\right|$

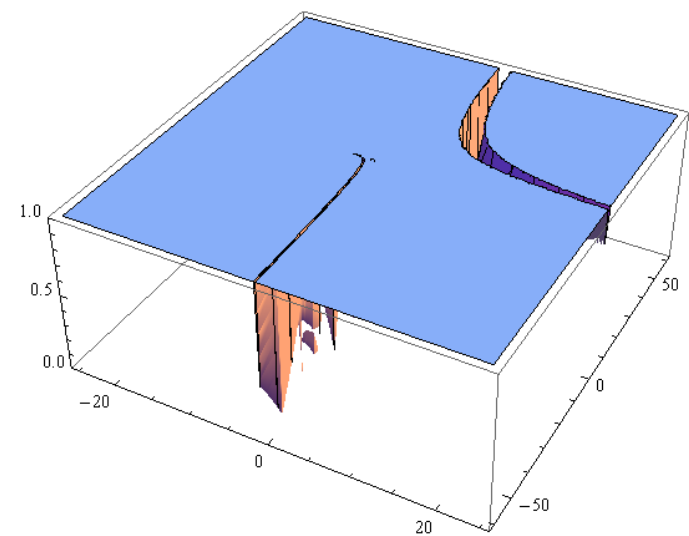

(d) $\mid O_{p}^{\prime}\left(s_{6}, \alpha, \beta\right)$

Figure 2: Stability functions for strange fixed points

Lemma 4.2. Points $z=0$ and $z=\infty$, associated to the roots of $p(z)$, are fixed points of $O_{p}(z, \alpha, 0)$. In addition, the number of strange fixed points is five,

$$
\begin{aligned}
z & =1, \text { if } \alpha \neq-3 \pm i, \\
s_{1}(\alpha) & =-\frac{1}{4} A(\alpha)+\frac{1}{4}(-5-2 \alpha)-\frac{\sqrt{1-\frac{35}{A(\alpha)}+8 \alpha-\frac{34 \alpha}{A(\alpha)}+2 \alpha^{2}-\frac{8 \alpha^{2}}{A(\alpha)}}}{2 \sqrt{2}} \\
s_{2}(\alpha) & =-\frac{1}{4} A(\alpha)+\frac{1}{4}(-5-2 \alpha)+\frac{\sqrt{1-\frac{35}{A(\alpha)}+8 \alpha-\frac{34 \alpha}{A(\alpha)}+2 \alpha^{2}-\frac{8 \alpha^{2}}{A(\alpha)}}}{2 \sqrt{2}} \\
s_{3}(\alpha) & =\frac{1}{4} A(\alpha)+\frac{1}{4}(-5-2 \alpha)-\frac{\sqrt{1+\frac{35}{A(\alpha)}+8 \alpha+\frac{34 \alpha}{A(\alpha)}+2 \alpha^{2}+\frac{8 \alpha^{2}}{A(\alpha)}}}{2 \sqrt{2}} \\
s_{4}(\alpha) & =\frac{1}{4} A(\alpha)+\frac{1}{4}(-5-2 \alpha)+\frac{\sqrt{1+\frac{35}{A(\alpha)}+8 \alpha+\frac{34 \alpha}{A(\alpha)}+2 \alpha^{2}+\frac{8 \alpha^{2}}{A(\alpha)}}}{2 \sqrt{2}}
\end{aligned}
$$

where $A(\alpha)=\sqrt{-7-4 \alpha}$, except in the following cases:

i) If $\alpha=-5-\sqrt{3}$, then $s_{1}=s_{2}=1$ and there are three different strange fixed points;

ii) If $\alpha=-5+\sqrt{3}$, then $s_{3}=s_{4}=1$ and there are also three different strange fixed points; 
iii) If $\alpha=-1 \pm i$, then $s_{1}=s_{2}=-1$ and there exist four strange fixed points;

iv) If $\alpha=-2$, then only strange fixed point is $z=1$.

v) If $\alpha=-3 \pm i$, then $z=1$ is not a fixed point, so there are only four.

Moreover, it can be proved that $s_{1}(\alpha)=\frac{1}{s_{2}(\alpha)}$ and $s_{3}(\alpha)=\frac{1}{s_{4}(\alpha)}$.

Respect to the asymptotical behavior of these fixed points, the stability function of each of them have been calculated and represented graphically in Figure 3. In it, three conical regions can be observed whose height represents the value of $\left|O_{p}^{\prime}(z, \alpha, 0)\right|$ on them and their basin are the complex values of parameter $\alpha$ where some of the strange fixed points are attractive. From left to right, the stability regions displayed are $\left|O_{p}^{\prime}\left(s_{1}, \alpha, 0\right)\right|=\left|O_{p}^{\prime}\left(s_{2}, \alpha, 0\right)\right|,\left|O_{p}^{\prime}(1, \alpha, 0)\right|$ and $\left|O_{p}^{\prime}\left(s_{3}, \alpha, 0\right)\right|=\left|O_{p}^{\prime}\left(s_{4}, \alpha, 0\right)\right|$. Out of these regions, all the strange fixed points are repulsive and, if the convergence of the method lead us to a fixed point, it will be a solution of our problem.

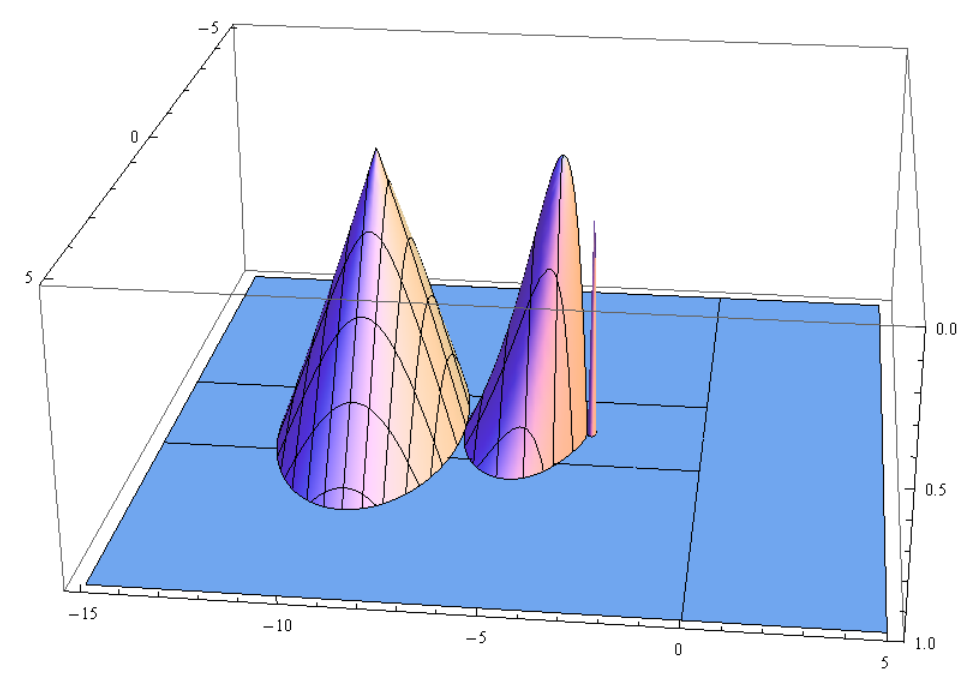

Figure 3: Stability functions of strange fixed points

However, it is possible that the convergence of the iterative schemes lead us to other attracting elements, such as periodic orbits. In order to detect this kind of behavior, the analysis of the critical points is necessary.

Critical points can be obtained by solving the equation $O_{p}^{\prime}(z, \alpha, 0)=0$. The roots of this equation are $z=0, z=\infty$, and also the free critical points, that are described in the following result.

Lemma 4.3. The free critical points of $O_{p}(z, \alpha, 0)$ are

$$
\begin{aligned}
& c r_{1}(\alpha)=\frac{1}{2}(-2-\alpha-\sqrt{\alpha} \sqrt{4+\alpha}) \\
& \operatorname{cr}_{2}(\alpha)=\frac{1}{2}(-2-\alpha+\sqrt{\alpha} \sqrt{4+\alpha}) \\
& \operatorname{cr}_{3}(\alpha)=\frac{-20-18 \alpha-6 \alpha^{2}-\alpha^{3}-\sqrt{\alpha} \sqrt{80+148 \alpha+128 \alpha^{2}+56 \alpha^{3}+12 \alpha^{4}+\alpha^{5}}}{4\left(5+4 \alpha+\alpha^{2}\right)}, \\
& \operatorname{cr}_{4}(\alpha)=\frac{-20-18 \alpha-6 \alpha^{2}-\alpha^{3}+\sqrt{\alpha} \sqrt{80+148 \alpha+128 \alpha^{2}+56 \alpha^{3}+12 \alpha^{4}+\alpha^{5}}}{4\left(5+4 \alpha+\alpha^{2}\right)}
\end{aligned}
$$

except in the following cases: 
i) If $\alpha=-4, c r_{1}=c r_{2}=c r_{3}=c r_{4}=1$ is the unique free critical point, that is also a superattracting fixed point.

ii) For $\alpha=-2, O_{p}^{\prime}(z,-2,0)=4 z^{3}$, and there not exist free critical points.

iii) When $\alpha=-3 \pm i$, the rational operator of the class is simplified and the only free fixed points are $c r_{1}(\alpha)$ and $\mathrm{cr}_{2}(\alpha)$.

iv) If $\alpha=-1 \pm i$, again the rational operator of the class is simplified and the only free fixed points are $c r_{1}(\alpha)$ and $\mathrm{cr}_{2}(\alpha)$.

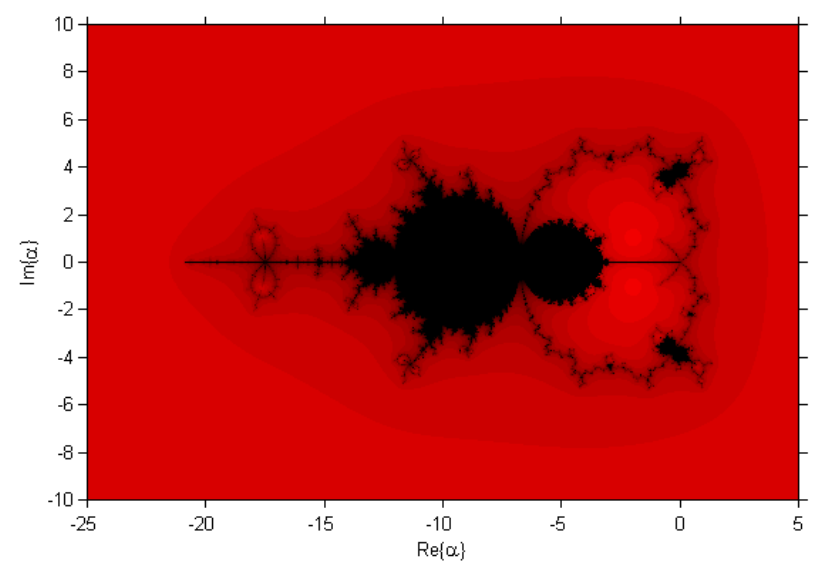

Figure 4: Parameter plane with $z_{0}=c r_{3}(\alpha)$.

Let us also remark that critical points satisfy $c r_{1}(\alpha)=\frac{1}{c r_{2}(\alpha)}$ and $c r_{3}(\alpha)=\frac{1}{c r_{4}(\alpha)}$. Moreover, $c r_{1}(\alpha)$ and $\mathrm{cr}_{2}(\alpha)$ are preperiodical points, as they are preimages of the strange fixed point $z=1$, $O_{p}\left(c r_{1}(\alpha), \alpha, 0\right)=O_{p}\left(c r_{2}(\alpha), \alpha, 0\right)=1$.

The dynamical behavior of operator 4.3 is globally observed in the parameter plane and it depends on the value of the parameter $\alpha$. It is obtained by applying the operator on an independent free critical point as initial estimation and coloring the point of the plane corresponding to the value of $\alpha$ depending on whether this critical goes to the basin of attraction of 0 or $\infty$ (red color) or to another unknown basin of attraction (black color). Moreover, each point of the parameter plane is associated with a complex value of $\alpha$, i.e., with an element of the family of iterative methods. Every value of parameter $\alpha$ belonging to the same connected component of the parameter space gives rise to subsets of elements of (4.2) with equivalent dynamical behavior. We have used the software presented in [20] with a mesh of $1000 \times 1000$ points, a maximum number of iterations of 200 and a tolerance of $10^{-3}$.

As it has been previously stated, free critical points $c r_{1}(\alpha)$ and $c r_{2}(\alpha)$ are preimages of the strange fixed point $z=1$, so there is no sense in analyzing its associated parameter space, as the information to be obtained is reflected in the stability function of $z=1$. On the other hand, we know that $c r_{3}(\alpha)=\frac{1}{c r_{4}(\alpha)}$. Then, there exist only one independent free critical point, whose associated parameter space can be seen in Figure 4. This figure is centered in a black region that corresponds mostly with the basins of the three cones that define the stability functions of the strange fixed points (see Figure 3). The rest of black areas are associated with other attracting behaviors, as of periodic orbits of different periods. In order to fully interpret the obtained parameter plane, we draw some dynamical planes corresponding to selected values of parameter $\alpha$ in these regions.

These dynamical planes have been also obtained by using the software included in [20, implemented in Matlab by using a mesh of $400 \times 400$, a maximum number of iterations of 40 and $10^{-3}$ as a tolerance. 


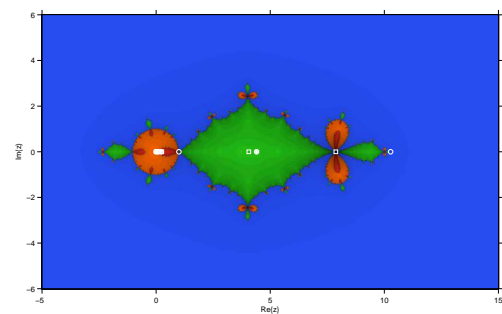

(a) $\alpha=-10$

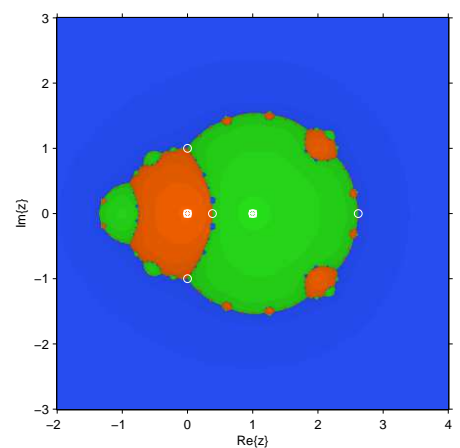

(c) $\alpha=-4$

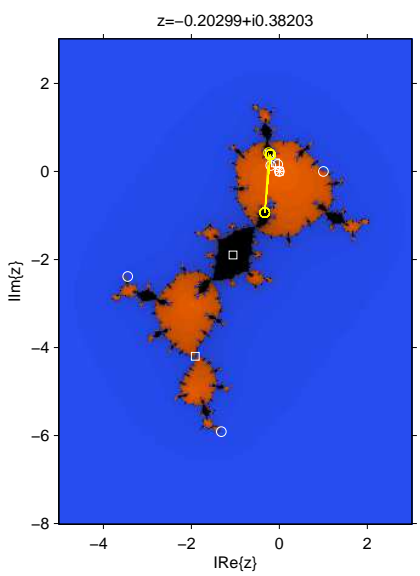

(e) $\alpha=4 i$

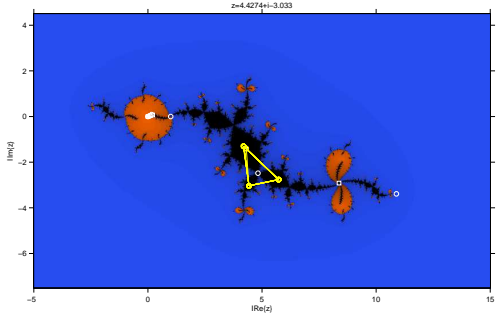

(b) $\alpha=-10.48+2.88 i$

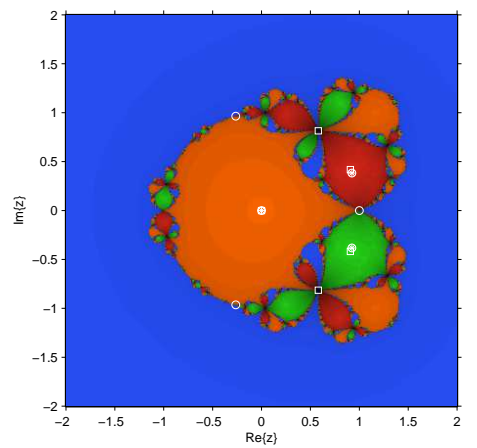

(d) $\alpha=-3.16$

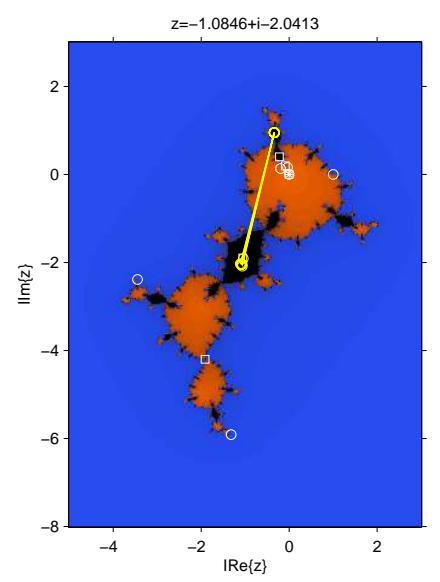

(f) $\alpha=4 i$

Figure 5: Dynamical planes with unstable behavior

The colors used also give us important information: orange regions are the basins of attraction of the fixed point 0 ; blue regions correspond to the basin of the infinity and the area of convergence of other strange fixed points are shown in other colors. Moreover, black regions are associated to attractive periodic orbits.

In Figure 5 , some non-desirable behaviors appear, corresponding to different black areas of parameter plane (Figure 4). The first one (Figure 5a) corresponds to the left big black region, where strange fixed points $s_{1}(\alpha)$ and $\left.s_{(} \alpha\right)$ are simultaneously attractive. Their basins of attraction are showed in green color different from the ones of $z=0$ and $z=\infty$. In an upper bulb of this region, for $\alpha=-10.48+2.88 i$, we found that the associated dynamical plane (in Figure $5 \mathrm{~b}$ includes the basin of attraction of a periodic orbit of period 3; this fact is specially important as we know, by Sharkovski's Theorem [21, that this implies the existence of periodic orbits of any period. Moreover, the superattracting character of $z=1$ can be observed in Figure $5 \mathrm{c}$ for $\alpha=-4$, where three basins of attraction appear. Close to this value, 


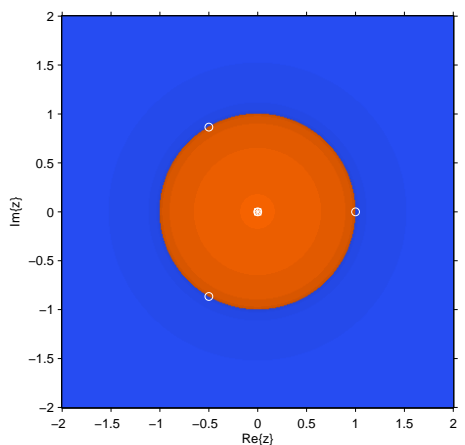

(a) $\alpha=-2$

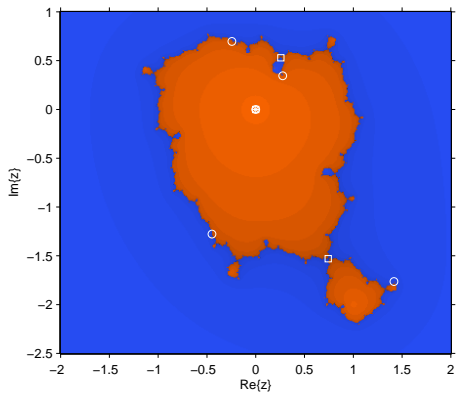

(c) $\alpha=-3+i$

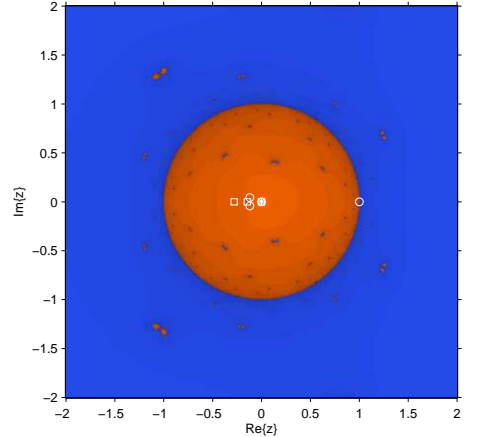

(b) $\alpha=5$

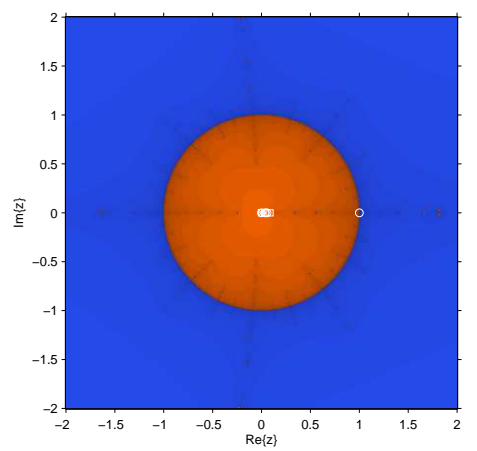

(d) $\alpha=-25$

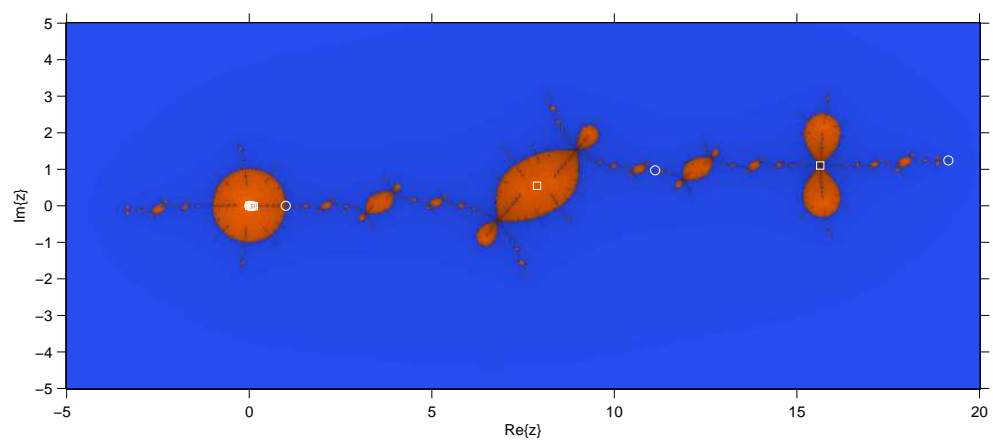

(e) $\alpha=-17.7-1.1 i$

Figure 6: Dynamical planes with stable behavior

at $\alpha=-3.16$, we find that strange fixed points $s_{3}(\alpha)$ and $s_{4}(\alpha)$ are simultaneously attracting, so four basins of attraction are observed. Finally, in Figures $5 \mathrm{e}$ and $5 \mathrm{ft}$ two different attracting 2-periodic orbits are shown for $\alpha=4 i$.

Finally, let us remark that the red region in the parameter space corresponds to dynamical planes where the only attractive points are 0 and $\infty$, that is, the points associated to the roots of the polynomial. This is the region with good numerical behavior. The associated dynamical planes for this region are similar to the one of Newton's scheme and can be seen in Figure6. The first one corresponds to $\alpha=-2$; let us remember that, for this value of the parameter, the rational operator is $z^{4}$ and the method satisfies Cayley's test. This is the reason why the dynamical plane is the same as Newton's one, but with fourthorder of convergence. The rest of figures included show different stable behavior with connected or 
disconnected basins of attraction.

\section{Numerical experiments}

In this section, we shall check the effectiveness of the newly proposed optimal methods. We employ some elements of the family that have proved to be specially stable in Section 4, specifically scheme (3.8) (for $\left.\alpha=-\frac{7}{4}\right)$, (3.10) for $\left(\alpha=1, H^{\prime \prime \prime}(1)=-57\right)$ and $\left(\alpha=-1, H^{\prime \prime \prime}(1)=9\right)$ denoted by $O M_{4}^{1}, O M_{4}^{2}$ and $O M_{4}^{3}$ respectively, to solve nonlinear equations given in Table 1. We compare them with method (9) proposed by Cordero et al. [10] (called by $A M_{4}$ ), an iterative expression (28) of Chun [8], denoted by $\left(C M_{4}\right)$ and method (9) developed by Chun and Ham 9 , denoted by $\left(C_{4}\right)$. In addition, King 7 proposed an optimal family of fourth-order methods (in expression (3.4)). From this family, we choose two particulars methods, for $\alpha=-1$ and $\alpha=-2$ (Ostrowski's method) denoted by $\left(K M_{4}\right)$ and $\left(O S_{4}\right)$, respectively.

In Table 2, we have also displayed the absolute errors in the function $f(x)$ (i.e. $\left|f\left(x_{n}\right)\right|$ ) for first four consecutive approximations and the meaning of $(A e-h)$ is $\left(A \times 10^{-h}\right)$. Further, in Table 3 , we mentioned the necessary number of iterations are required to achieve the desire accuracy to the corresponding zeros of the functions $f_{1}(x)-f_{5}(x)$. Further, we also want to verify the theoretical order of convergence which is proven in section 2. Therefore, we could calculate the computational order of convergence (see [22]), by using the following formula

$$
\rho \approx \frac{\ln \left|\left(x_{n+1}-\xi\right) /\left(x_{n}-\xi\right)\right|}{\ln \left|\left(x_{n}-\xi\right) /\left(x_{n-1}-\xi\right)\right|} .
$$

But, the main drawback of this COC is that it involves the exact root $\xi$ and there are many real situations in which the exact root is not known in advance. To overcome this problem, Cordero and Torregrosa in [23], redefined the definition of $\mathrm{COC}$, which does not require the exact root as follows:

$$
\rho \approx \frac{\ln \left|x_{n+1}-x_{n}\right| /\left|x_{n}-x_{n-1}\right|}{\ln \left|x_{n}-x_{n-1}\right| /\left|x_{n-1}-x_{n-2}\right|},
$$

denoted by ACOC. All computations have been performed using the programming package Mathematica 9 with multiple precision arithmetic. We use $\epsilon=10^{-34}$ as a tolerance error. The following stopping criteria are used for computer programs:

$(i)\left|x_{n+1}-x_{n}\right|<\epsilon$ and $(i i)\left|f\left(x_{n+1}\right)\right|<\epsilon$.

In Table 2 we represent highlighted in bold the best results for each example and iteration. It is

Table 1: Test problems

\begin{tabular}{|cc|}
\hline$f(x)$ & $\xi$ \\
\hline$f_{1}(x)=e^{-x}+\cos x$ & 1.7461395304080124176507030889537802 \\
$f_{2}(x)=\tan ^{-1}\left(x^{2}-x\right)$ & 1 \\
$f_{3}(x)=x^{3}-30 x+5$ & 0.16682141791816451151054900720209898 \\
$f_{4}(x)=(x-2)^{2}-\log x-33 x$ & 36.989473582944669865344734734912736 \\
$f_{5}(x)=10 x e^{-x^{2}}-1$ & 1.6796306104284499406749203388379704 \\
\hline
\end{tabular}

observed that proposed methods are competitive in comparison with excellent schemes as Chun's and Ostrowski's ones.

\section{Conclusions}

In this paper, we have obtained a wide general optimal class of fourth-order methods which are free from second-order derivative. We can easily obtain various new methods by considering some particular values of disposable parameters in schemes (3.8) and (3.10). Further on, we can also develop various new and existing families of methods just by considering different types of weight functions. Furthermore, we 
Table 2: Comparison of fourth-order optimal methods with respect to absolute error in function $f(x)$

\begin{tabular}{|c|c|c|c|c|c|c|c|c|c|c|}
\hline$f(x)$ & $x_{0}$ & $|f(x)|$ & $A M_{4}$ & $C M_{4}$ & $\mathrm{CH}_{4}$ & $K M_{4}$ & $O S_{4}$ & $O M_{4}^{1}$ & $O M_{4}^{2}$ & $O M_{4}^{3}$ \\
\hline \multirow{4}{*}{$f_{1}(x)$} & \multirow{4}{*}{2.0} & $\left|f\left(x_{1}\right)\right|$ & $3.7 \mathrm{e}-4$ & $3.0 \mathrm{e}-4$ & $3.0 \mathrm{e}-4$ & $3.0 \mathrm{e}-4$ & $1.8 \mathrm{e}-4$ & $1.8 \mathrm{e}-4$ & $2.4 \mathrm{e}-6$ & $1.5 \mathrm{e}-4$ \\
\hline & & $\left|f\left(x_{2}\right)\right|$ & $3.7 e-16$ & $1.4 \mathrm{e}-16$ & $1.5 \mathrm{e}-16$ & $1.5 \mathrm{e}-16$ & $1.4 \mathrm{e}-17$ & $1.3 \mathrm{e}-17$ & $4.5 \mathrm{e}-25$ & $5.6 \mathrm{e}-18$ \\
\hline & & $\left|f\left(x_{3}\right)\right|$ & $3.7 e-64$ & $7.5 \mathrm{e}-66$ & $8.3 e-66$ & $9.3 e-66$ & $5.3 e-70$ & $3.3 \mathrm{e}-70$ & $4.8 \mathrm{e}-100$ & $1.2 \mathrm{e}-71$ \\
\hline & & $\left|f\left(x_{4}\right)\right|$ & $3.9 \mathrm{e}-256$ & $5.7 \mathrm{e}-263$ & $8.6 \mathrm{e}-263$ & $1.3 \mathrm{e}-262$ & $1.0 \mathrm{e}-279$ & $1.5 \mathrm{e}-280$ & $6.8 \mathrm{e}-400$ & $2.4 \mathrm{e}-286$ \\
\hline \multirow{4}{*}{$f_{2}(x)$} & \multirow{4}{*}{1.6} & $\left|f\left(x_{1}\right)\right|$ & $2.1 \mathrm{e}-2$ & $2.1 \mathrm{e}-2$ & $2.1 \mathrm{e}-2$ & $2.1 \mathrm{e}-2$ & $2.0 \mathrm{e}-2$ & $2.0 \mathrm{e}-2$ & $1.9 \mathrm{e}-2$ & $2.0 \mathrm{e}-2$ \\
\hline & & $\left|f\left(x_{2}\right)\right|$ & $1.1 \mathrm{e}-6$ & $7.8 \mathrm{e}-7$ & $7.8 \mathrm{e}-7$ & $7.9 \mathrm{e}-7$ & $2.6 \mathrm{e}-7$ & $2.4 \mathrm{e}-7$ & $3.8 \mathrm{e}-9$ & $1.4 \mathrm{e}-7$ \\
\hline & & $\left|f\left(x_{3}\right)\right|$ & $6.5 \mathrm{e}-24$ & $1.2 \mathrm{e}-24$ & $1.3 \mathrm{e}-24$ & $1.3 \mathrm{e}-24$ & $6.2 \mathrm{e}-27$ & $4.4 \mathrm{e}-27$ & $1.8 \mathrm{e}-34$ & $3.5 \mathrm{e}-28$ \\
\hline & & $\left|f\left(x_{4}\right)\right|$ & $7.9 \mathrm{e}-93$ & $7.5 \mathrm{e}-96$ & $8.2 \mathrm{e}-96$ & $9.1 \mathrm{e}-96$ & $2.0 \mathrm{e}-105$ & $4.6 \mathrm{e}-106$ & $8.0 \mathrm{e}-136$ & $1.2 \mathrm{e}-110$ \\
\hline \multirow{4}{*}{$f_{3}(x)$} & \multirow{4}{*}{1.0} & $\left|f\left(x_{1}\right)\right|$ & $6.6 \mathrm{e}-2$ & $5.8 \mathrm{e}-2$ & $5.8 \mathrm{e}-2$ & $5.9 \mathrm{e}-2$ & $4.4 \mathrm{e}-2$ & $4.3 \mathrm{e}-2$ & $2.0 \mathrm{e}-2$ & $4.0 \mathrm{e}-2$ \\
\hline & & $\left|f\left(x_{2}\right)\right|$ & $4.2 \mathrm{e}-13$ & $2.4 \mathrm{e}-13$ & $2.5 \mathrm{e}-13$ & $2.5 \mathrm{e}-13$ & $7.8 \mathrm{e}-14$ & $7.5 \mathrm{e}-14$ & $\mathbf{3 . 5} \mathrm{e}-15$ & $5.3 \mathrm{e}-14$ \\
\hline & & $\left|f\left(x_{3}\right)\right|$ & $6.5 \mathrm{e}-58$ & $7.4 \mathrm{e}-59$ & $7.9 \mathrm{e}-59$ & $8.5 \mathrm{e}-59$ & $8.0 \mathrm{e}-61$ & $6.5 \mathrm{e}-61$ & $3.1 e-66$ & $1.6 \mathrm{e}-61$ \\
\hline & & $\left|f\left(x_{4}\right)\right|$ & $3.8 \mathrm{e}-237$ & $6.3 e-241$ & $8.4 \mathrm{e}-241$ & $1.1 \mathrm{e}-240$ & $8.5 \mathrm{e}-249$ & $3.8 \mathrm{e}-249$ & $1.9 \mathrm{e}-270$ & $1.5 \mathrm{e}-251$ \\
\hline \multirow{4}{*}{$f_{4}(x)$} & \multirow{4}{*}{36} & $\left|f\left(x_{1}\right)\right|$ & $3.4 \mathrm{e}-3$ & $2.5 \mathrm{e}-3$ & $2.5 \mathrm{e}-3$ & $2.5 \mathrm{e}-3$ & $7.8 \mathrm{e}-4$ & $7.2 \mathrm{e}-4$ & $4.7 e-4$ & $3.3 \mathrm{e}-4$ \\
\hline & & $\left|f\left(x_{2}\right)\right|$ & $2.0 \mathrm{e}-19$ & $4.3 \mathrm{e}-20$ & $4.4 \mathrm{e}-20$ & $4.5 \mathrm{e}-20$ & $1.5 \mathrm{e}-22$ & $9.5 \mathrm{e}-23$ & $1.0 \mathrm{e}-23$ & $2.5 \mathrm{e}-24$ \\
\hline & & $\left|f\left(x_{3}\right)\right|$ & $2.5 \mathrm{e}-84$ & $4.1 \mathrm{e}-87$ & $4.5 \mathrm{e}-87$ & $4.9 \mathrm{e}-87$ & $1.9 \mathrm{e}-97$ & $3.0 \mathrm{e}-98$ & $\mathbf{2 . 0 e}-102$ & $7.4 \mathrm{e}-105$ \\
\hline & & $\left|f\left(x_{4}\right)\right|$ & $6.6 \mathrm{e}-344$ & $3.4 \mathrm{e}-355$ & $4.7 \mathrm{e}-355$ & $6.6 \mathrm{e}-355$ & $5.2 \mathrm{e}-397$ & $2.8 \mathrm{e}-400$ & $3.0 \mathrm{e}-417$ & $5.9 \mathrm{e}-427$ \\
\hline \multirow{4}{*}{$f_{5}(x)$} & \multirow{4}{*}{1.1} & $\left|f\left(x_{1}\right)\right|$ & $2.9 \mathrm{e}-2$ & $2.8 \mathrm{e}-2$ & $2.8 \mathrm{e}-2$ & $2.8 \mathrm{e}-2$ & $2.8 \mathrm{e}-2$ & $2.8 \mathrm{e}-2$ & $2.8 \mathrm{e}-2$ & $2.8 \mathrm{e}-2$ \\
\hline & & $\left|f\left(x_{2}\right)\right|$ & $9.9 \mathrm{e}-8$ & $7.2 \mathrm{e}-8$ & $7.2 \mathrm{e}-8$ & $7.2 \mathrm{e}-8$ & $2.2 \mathrm{e}-8$ & $2.0 \mathrm{e}-8$ & $1.7 \mathrm{e}-8$ & $1.1 \mathrm{e}-8$ \\
\hline & & $\left|f\left(x_{3}\right)\right|$ & $1.6 \mathrm{e}-29$ & $3.3 e-30$ & $3.3 \mathrm{e}-30$ & $3.3 e-30$ & $8.7 e-33$ & $5.4 \mathrm{e}-33$ & $1.5 \mathrm{e}-33$ & $2.1 \mathrm{e}-34$ \\
\hline & & $\left|f\left(x_{4}\right)\right|$ & $1.0 \mathrm{e}-116$ & $1.5 \mathrm{e}-119$ & $1.4 \mathrm{e}-119$ & $1.4 \mathrm{e}-119$ & $2.1 \mathrm{e}-130$ & $2.9 \mathrm{e}-131$ & $8.6 e-134$ & $3.7 \mathrm{e}-137$ \\
\hline
\end{tabular}
Bold-face numbers denote the least error among the listed methods. 
Table 3: Comparison of fourth-order optimal methods with respect to number of iterations

\begin{tabular}{|cccccccccc|}
\hline$f(x)$ & $x_{0}$ & $A M_{4}$ & $C M_{4}$ & $C H_{4}$ & $K M_{4}$ & $O S_{4}$ & $O M_{4}^{1}$ & $O M_{4}^{2}$ & $O M_{4}^{3}$ \\
\hline$f_{1}(x)$ & 2.0 & 4 & 4 & 4 & 4 & 4 & 4 & 4 & 4 \\
$f_{2}(x)$ & 1.6 & 5 & 5 & 5 & 5 & 5 & 5 & 4 & 5 \\
$f_{3}(x)$ & 1.0 & 4 & 4 & 4 & 4 & 4 & 4 & 4 & 4 \\
$f_{4}(x)$ & 36 & 4 & 4 & 4 & 4 & 4 & 4 & 4 & 4 \\
$f_{5}(x)$ & 1.1 & 5 & 5 & 5 & 5 & 5 & 5 & 4 & 4 \\
\hline
\end{tabular}

Table 4: Comparison of ACOC of fourth-order methods

\begin{tabular}{|cccccccccc|}
\hline$f(x)$ & $x_{0}$ & $A M_{4}$ & $C M_{4}$ & $C H_{4}$ & $K M_{4}$ & $O S_{4}$ & $O M_{4}^{1}$ & $O M_{4}^{2}$ & $O M_{4}^{3}$ \\
\hline$f_{1}(x)$ & 2.0 & 4.000 & 4.000 & 4.000 & 4.000 & 4.000 & 4.000 & 4.000 & 4.000 \\
$f_{2}(x)$ & 1.6 & 4.000 & 4.000 & 4.000 & 4.000 & 4.000 & 4.000 & 3.777 & 4.000 \\
$f_{3}(x)$ & 1.0 & 4.000 & 4.000 & 4.000 & 4.000 & 4.000 & 4.000 & 4.000 & 4.000 \\
$f_{4}(x)$ & 36 & 4.000 & 4.000 & 4.000 & 4.000 & 4.000 & 4.000 & 4.000 & 4.000 \\
$f_{5}(x)$ & 1.1 & 4.000 & 4.000 & 4.000 & 4.000 & 4.000 & 4.000 & 4.034 & 4.003 \\
\hline
\end{tabular}

also have shown that the well-known Ostrowski's method and King's family of fourth-order are special cases of our proposed class. Every member of our family requires only two evaluations of the function and one evaluations of its first-order derivative per full iteration. The dynamical analysis made allows us to select some elements of the families that are specially stable, rejecting those where pathological behaviors appear, such as attracting strange fixed points or periodic orbits. Finally, we conclude from Tables 2 to 4 that our proposed methods are equally or better competitive with the existing classical Ostrowski's and King's methods and other recognized efficient methods available in the literature.

\section{References}

[1] J.F. Traub, Iterative methods for the solution of equations, Prentice-Hall, Englewood Cliffs, New York, 1964.

[2] M.S. Petković, B. Neta, L.D. Petković, J. Džunić, Multipoint methods for solving nonlinear equations, Academic Press, Amsterdam, 2013.

[3] A.M. Ostrowski, Solutions of Equations and System of Equations, Academic Press, New York, 1960.

[4] H.T. Kung, J. F. Taub, Optimal order of one-point and multi-point iteration, J. ACM. 21 (1974) 643-651.

[5] P. Jarratt, Some fourth-order multipoint iterative methods for solving equations, Math. Comput. 20 (1966) 434-437.

[6] P. Jarratt, Some efficient fourth-order multipoint methods for solving equations, BIT (1969) 119-124.

[7] R. F. King, A family of fourth order methods for nonlinear equations, SIAM J. Numer. Anal. 10 (1973) 876-879.

[8] C. Chun, Some variants of King's fourth-order family of methods for nonlinear equations, Appl. Math. Comput. 190 (2007) 57-62. 
[9] C. Chun, Y.M. Ham, Some fourth-order modifications of Newton's method, Appl. Math. Comput. 197 (2008) 654-658.

[10] A. Cordero, J.L. Hueso, E. Martínez, J.R. Torregrosa, New modifications of Potra-Pták's method with optimal fourth and eighth orders of convergence, J. Comput. Appl. Math. 234 (2010) 2969-2976.

[11] J.R. Sharma, R.K. Guha, Second-derivative free methods of third and fourth order for solving nonlinear equations, Int. J. Comput. Math. 88 (2010) 163-170.

[12] V. Kanwar, R. Behl, K.K. Sharma, Simply constructed family of a Ostrowski's method with optimal order of convergence, Comput. Math. Appl. 62 (2011) 4021-4027.

[13] M. Sharifi, D.K.R. Babajee, F. Soleymani, Finding the solution of nonlinear equations by a classof optimal methods, Comput. Math. Appl. 63 (4) (2012) 764-774.

[14] F. Soleymani, S. K. Khattri, S. K. Vanani, Two new classes of optimal Jarratt-type fourthorder methods, Appl. Math. Lett. 25(5) (2012) 847-853.

[15] R. Behl, V. Kanwar, K.K. Sharma, Optimal equi-scaled families of Jarratt's method, Int. J. Comput. Math. 90(2) (2013) 408-422.

[16] P. Blanchard, Complex Analytic Dynamics on the Riemann Sphere, Bull. of the AMS 11(1) (1984) 85-141.

[17] S. Artidiello, Diseño, implementación y convergencia de métodos iterativos para resolver ecuaciones y sistemas no lienales utilizando funciones peso, Ph.D. Thesis, Universitat Politècnica de València, 2014.

[18] P. Blanchard, The dynamics of Newton's method. Proc. Symp. Appl. Math. 49 (1994) 139-154.

[19] D.K.R. Babajee, A. Cordero, J.R. Torregrosa, Study of iterative methods through the Cayley Quadratic Test, J. Comput. Appl. Math. doi: 10.1016/j.cam.2014.09.020.

[20] F. Chicharro, A. Cordero, J.R. Torregrosa, Drawing dynamical and parameter planes of iterative families and methods, The Sci. World J. 2013 (2013) Article ID 780153.

[21] R.L. Devaney, The Mandelbrot Set, the Farey Tree and the Fibonacci sequence. Amer. Math. Monthly 106(4) (1999) 289-302.

[22] S. Weerakoon, T.G.I. Fernando, A variant of Newton's method with accelerated third-order convergence, Appl. Math. Lett. 13 (2000) 87-93.

[23] A. Cordero, J.R. Torregrosa, Variants of Newton's Method using fifth-order quadrature formulas, Appl. Math. Comput. 190 (2007) 686-698. 